\title{
Discussion on the Relevant Factors of General Surgery Incision Infection and Prevention Methods
}

\author{
Baotao $\mathrm{Jin}^{1 *}$, Changsheng $\mathrm{Lv}^{1}$ and $\mathrm{Hu} \mathrm{Kai}^{1}$ \\ ${ }^{1}$ Department of General Surgery, Jingzhou Second Renmin Hospital, Jingzhou, Hubei Province,434000, China \\ Corresponding E-mail:270882285@qq.com
}

\begin{abstract}
There are many reasons that can lead to incision infection of general surgical patients. The main reasons include weight, age, body albumin level, surgical time, observation ward, etc. This paper analyzes the clinic data of patients with incision infection after general surgery based on clinic practice and study on the reasons that have impact on general surgical incision infection and gives relevant prevention countermeasures.
\end{abstract}

Keywords. General surgery department, incision infection, prevention countermeasures.

\section{Introduction}

The department of general surgery, which is a clinical department with surgery as its main treatment means, has significant curative effect in treating liver, intestines, stomach, vascular disease, biliary tract, thyroid and tumor etc. Wound infection is the factor which affects the curative effect of the department of general surgery most seriously. Besides, postoperative wound infection is one of the most common clinical complications in the department of general surgery. According to the incomplete data statistics, its incidence rate is $9.45 \% \sim 14.9 \%$, so it has become the most important factor influencing a patient's life safety [1]. Furthermore, there are numerous factors clinically causing a patient's post-operative wound infection. In order to analyze the factors causing a patient's wound infection.

\section{Data}

Case selection: 679 patients with surgical treatment at the department of general surgery in our hospital from January 2009 to December 2014, including 412 male patients and 267 female patients with their ages from 6 to 76 years old.

Diagnostic criteria: refer to the Diagnostic Criteria for Nosocomial Infection (Trial) issued by the Ministry of Health.

\section{Methods}

The retrospective investigation method was used. The investigation mainly included the pre-operative factors: patients' ages, combination of underlying diseases (anemia and diabetes mellitus), operation timing, season of operation, duration of operation, type of operation, suture by medical staff and post-operative hospitalization duration etc. In this study, the SPSS statistical software (18.0 version) was used to analyze and process data. Besides, as for the clinical data obeying normal distribution in this study, two independent samples $t$ were used for inspection; as for the enumeration data, chi-square was used for inspection. The result of study was $\mathrm{P}<0.005$, so the difference is of statistical significance. 


\section{Result}

The infection factors of operative incision of the department of general surgery are related to ages, time of operation, property of surgery and type of incision to some degree, as shown in Table 1.

Table 1. Analysis on predisposing factors of operative incision of the department of general surgery.

\begin{tabular}{|c|c|c|c|c|c|}
\hline & $\begin{array}{c}\text { Number of } \\
\text { operations }\end{array}$ & $\begin{array}{c}\text { Number of } \\
\text { infected } \\
\text { cases }\end{array}$ & Infection rate (\%) & $\chi 2$ value & P \\
\hline Age & & & & 4.624 & $<0.05$ \\
\hline$<30$ years old & 156 & 5 & 3.21 & & \\
\hline $30 \sim 60$ years old & 342 & 8 & 2.34 & & \\
\hline$>60$ years old & 181 & 10 & 5.52 & & $<0.01$ \\
\hline Time of operation & & & & 17.628 & \\
\hline$<120$ min & 463 & 6 & 0.88 & & $<0.01$ \\
\hline$\geq 120$ min & 216 & 17 & 7.87 & & \\
\hline Type of incision & & & & 14.629 & \\
\hline Type I & 346 & 5 & 1.45 & & \\
\hline Type II & 243 & 10 & 4.12 & & \\
\hline Type III & 90 & 8 & 8.89 & & \\
\hline
\end{tabular}

\section{Discussion}

Operative wound infection is one of the main infections among nosocomial infections. About $2 \% \sim 5 \%$ of patients receiving abdominal external aseptic operation and $20 \%$ of patients receiving intra-abdominal operation will suffer wound infection. As indicated in related data, about 500,000 patients suffer the infection of surgical site in America every year. Besides, compared with the patients without infection, those with infections are affected in their death danger, hospitalization time and cost and they also probably would require hospitalization again. In addition, the infection rate increases with the degree of seriousness of a disease, because a large operation will increase the time of operation and the invasion of bacteria. Then, a patient's skin and mucosa are cut up during an operation, so their organism lacks the defense against bacteria, raising the infection rate. One of the most common infections is the infection of surgical incision by the department of general surgery, which concerns a patient and all links during an operation [2-4].

\subsection{Factors causing infection of surgical incision by the department of general surgery}

It can be found through the above-mentioned study that the infection of operative wound by the department of general surgery is related to the following factors: (1) a patient' $s$ age: the degradation of intracorporeal organs of the aged and lowered immunity will lower a patient' s wound healing ability and thus result in wound infection. Due to the not mature immune system, a minor patient has a poor resistance against the trauma of wound. Therefore, more clinical attention shall be paid to the old or young patients so as to avoid the deterioration of their wounds. (2) Is a patient obese: an obese patient' s high content of internal fact will result in his/her weakened immunity, which will easily cause wound infection. (3) Does a patient have the medical history of diabetes: the granulation tissue formed in a diabetic patient' s body prolongs the duration of wound healing and provides a favorable condition for the bacterial infection of wound. For such patients, insulin treatment is required before an operation to alleviate a patient' s symptom of diabetes and keep his/her glucostasis [5-7]. (4) Type of operation: a type II incision is a hazardous factor leading to infection mainly due to the not strict operation of the surgical process. During a surgery of the department of general surgery, the operating room shall 
strictly follow the requirement of aseptic technique, strengthen the awareness of aseptic technique and thus reduce the possibility of wound infection of a patient. (5) Time of operation: the duration of operation is largely related to a patient' $s$ wound infection. A longer duration of operation requires a longer exposure of a patient' $s$ wound, so the possibility of infection will increase accordingly. Clinically, it is required to conduct corresponding clinical inspections before an operation, determine the therapeutic schedule, accelerate the process of operation, shorten the duration of operation, reduce the a patient' s parts exposed and guarantee strict sterile operation.

\subsection{Strategies for preventing infection of surgical incision by the department of general surgery}

An incision of surgery of the department of general surgery has a high risk of infection and there are abundant complicated causes causing an infection, so targeted protective measures are required during the perioperative period to guarantee the smooth post-operative recovery of a patient and lower the impact of incision infection. (1) As for an old patient, it is required to assess his/her overall physical condition firstly, strictly execute aseptic techniques during an operation and actively treat the complication; (2) As for a patient with a high body mass index, it is required to cut the subcutaneous tissue with one cut, avoid forcibly rubbing the fat layer, stop bleeding thoroughly, reserve no dead space during suture and flush the incision with stroke-physiological saline solution before suture; as for a patient with a thick fat layer, layered suture can be adopted and relaxation suture can also be conducted in a special condition. (3) The nutritional support must be in place. Albumin can be conveyed to a patient when necessary so as to increase his/her resistance. (4) Strengthen the technical operation level of medical staff and enhance the teamwork so as to guarantee the quality of operation and reduced duration of operation simultaneously. (5) Use an electrotome for cutting and coagulation in a scientific and effective way; avoid the excessive increase in the output frequency of an electrotome; in an operation involving fat and fascia tissue, impose certain tensile force to separate tissue and minimize the thermal energy contact between an electrotome and tissue so as to reduce the inactivation and liquefaction probabilities of tissue [8, 9]. Provide separate wards; separate patients with wound infection from those with aseptic operation; strengthen the management over ware environment; control the visitors; respectively arrange the family visit time and centralized dressing change time.

The existence of underlying diseases lowers the body immunity and resistance and increases the incision infection rate, such as merged diabetes mellitus. The hyperglycemia facilitate the bacterial growth in tissue and diabetic patients suffer the metabolic disorder at varying degrees, which is further aggregated by the stress response of an operation, so the bactericidal ability of their cells in their body is weakened to cause the poor tissue healing ability and thus to cause easy infection[10]. For this reason, ideally control the blood glucose and urine glucose with insulin during the perioperative period. Timely adjust the dosage of insulin after operation according to the metabolic disorder of blood glucose and the level of sensitivity for insulin. In addition, improve a patient's nutrition, preventively use antibiotics and select a proper anesthetic method and medicine to minimize the occurrence of complication and thus ensure that a patient can safely live through the period of operation.

\section{Conclusion}

All in all, the incision infection during the surgery of the department of general surgery is caused by multiple factors, so we should take measures from many aspects to reduce the incision infection, such as shortening the length of incision, strengthen aseptic technique of surgery and using antibiotics.

\section{References}

1. Ventral Hernia Outcome Collaborative, Mitchell TO1, Holihan JL2, Askenasy EP3, Greenberg JA4, Keith JN5, Martindale RG6, Roth JS7, Liang MK.Do risk calculators accurately predict surgical site occurrences?J Surg Res. 2016 Jun 1;203(1):56-63. 
2. Golub AV1, Kozlov RS2, Pleshkov VG1, Moskalev AP3, Alibegov RA4, Chelombit'ko MA5.Surgical Site Infections after Open Appendectomy and Effectiveness of Complex Approach to TheirPrevention. Khirurgiia (Mosk). 2016;(6):68-76.

3. Zhou ZY1, Liu YK1, Chen HL2, LiF.Prevention of Surgical Site Infection After Ankle Surgery Using Vacuum-Assisted Closure Therapy in High-Risk Patients With Diabetes.J Foot Ankle Surg. 2016 Jan-Feb;55(1):129-31.

4. Ngai IM1, Van Arsdale A, Govindappagari S, Judge NE, Neto NK, Bernstein J, Bernstein PS, Garry DJ.Skin for Prevention of Surgical Site Infection After Cesarean Delivery: A Randomized Controlled Trial.Obstet Gynecol. 2015 Dec;126(6):1251-7.

5. Kiernan M1. Prevention of surgical site infection: compliance is key.Br J Nurs. 2015 Sep 24-Oct 7;24(17):856.

6. Nodzo SR1.Commentary to "CDC Will Soon Issue Guidelines for the Prevention of Surgical Site Infection".Am J Orthop (Belle Mead NJ). 2015 Sep;44(9):E360.

7. Matsumoto M1, Shigemura K, Yamamichi F, Nakano Y, Tanaka K, Arakawa S, Fujisawa M.Prophylactic antibiotic for prevention of surgical site infection in urological laparoscopic surgeries.Kobe J Med Sci. 2012 May 24;57(4):E137-44.

8. Beckmann A1, Doebler Khttps://www.ncbi.nlm.nih.gov/pubmed/?term=Graf K[Author]\&cauthor=true\&cauthor_uid=21277786. Sternal surgical site infection prevention - is there any room for improvement?Eur J Cardiothorac Surg. 2011 Aug;40(2):347-51.

9. Matsumoto M1, Shigemura K, Yamamichi F, Nakano Y, Tanaka K, Arakawa S, Fujisawa M.Prophylactic antibiotic for prevention of surgical site infection in urological laparoscopic surgeries.Kobe J Med Sci. 2012 May 24;57(4):E137-44.

10. Weiser MC1, Moucha CS1.The Current State of Screening and Decolonization for the Prevention of Staphylococcus aureus Surgical SiteInfection After Total Hip and Knee Arthroplasty.J Bone Joint Surg Am. 2015 Sep 2;97(17):1449-58. 